\title{
Radiation pressure and pulsation effects on the Roche lobe
}

\author{
T. Dermine ${ }^{1}$, A. Jorissen ${ }^{1}$, L. Siess ${ }^{1}$, and A. Frankowski ${ }^{2}$ \\ 1 Institut d'Astronomie et d'Astrophysique, Université libre de Bruxelles, Faculté des Sciences, CP 226, Boulevard du Triomphe, \\ 1050 Bruxelles, Belgium \\ e-mail: tdermine@ulb.ac.be \\ 2 Department of Physics, Technion-Israel Institute of Technology, 32000 Haifa, Israel
}

Received 9 April 2009 / Accepted 15 August 2009

\begin{abstract}
Context. Several observational pieces of evidence indicate that specific evolutionary channels that involve Roche lobe overflow are not correctly accounted for by the classical Roche model.

Aims. We generalise the concept of the Roche lobe in the presence of extra forces (caused by radiation pressure or pulsations). By computing the distortion of the equipotential surfaces, we are able to evaluate the impact of these perturbing forces on the stability of Roche lobe overflow (RLOF).

Methods. Radiative forces are parametrised through the constant reduction factor that they impose on the gravitational force from the radiating star (neglecting any shielding in the case of high optical thickness). Forces imparted by pulsation are derived from the velocity profile of the wind that they trigger.

Results. We provide analytical expressions to compute the generalised Roche radius. Depending on the extra force, the Roche lobe radius may either stay unchanged, become smaller, or even become meaningless (in the presence of a radiatively- or pulsation-driven wind). There is little impact on the RLOF stability.
\end{abstract}

Key words. stars: binaries: general - stars: mass-loss - stars: winds, outflows

\section{Introduction}

The Roche model has been widely used to infer the outcome of binary star evolution. In this model, only the gravitational and centrifugal forces are accounted for to compute the equipotential surfaces. However, if other forces are present in the system, like those responsible for mass loss, they should be included in the description as well. The modification of the usual Roche model was first pointed out by Schuerman (1972) in the context of binary systems involving early-type main sequence stars with a strong wind. The idea was further explored in various directions by Kondo \& McCluskey (1976), Vanbeveren (1977, 1978), Friend \& Castor (1982), Djurasevic (1986), Zhou \& Leung (1988), Huang \& Taam (1990), Drechsel et al. (1995), Frankowski \& Tylenda (2001), Phillips \& Podsiadlowski (2002) and Owocki (2007). In the present paper, we investigate in a more systematic way the situations where the Roche model should be modified by considering the different physical processes driving stellar winds, that we briefly describe in Sect. 2.

The Roche model is generally used to answer two different questions, namely: (i) What is the flow geometry? (ii) Is the star filling its Roche lobe? The first question is related to the geometry of the equipotentials (and to the Coriolis force). One important modification of the equipotential geometry which can arise in the presence of an extra force pervading all space (like radiation pressure), is that the equipotentials open up in the direction of the external Lagrangian point, thus possibly allowing the matter ejected by the mass-losing component to form a circumbinary disc. This issue is important in the framework of binary evolution involving low- and intermediate-mass components where the formation of a circumbinary disc seems to be very common (see de Ruyter et al. 2006; Frankowski \& Jorissen 2007; Frankowski 2009).

The second question corresponds to the Roche-lobe overflow (RLOF) criterion, which involves the comparison of the Roche radius with the stellar radius. In the present paper we show how, depending on the physical process driving the wind, the Roche radius may either be unchanged with respect to the classical Roche model, become smaller, or even become meaningless. This will depend on the value of the extra force at the stellar surface (in contrast to the first question, an answer to which requires the knowledge of the force everywhere within the system). In a mass-losing star, the photospheric radius itself may become illdefined, thus complicating the use of the RLOF criterion (see Sect. 7.4).

Both issues (the equipotential geometry and the RLOF criterion) will be addressed in the present paper for the generalised Roche model when radiation pressure or pulsations play a role (Sect. 3). The way to correctly account for radiation pressure is discussed in Sect. 4. Typical values for the radiation pressure at the surface of various classes of stars are given in Sect. 5, and the corresponding shapes of the equipotentials are displayed in Sect. 6. A numerical fit to the Roche radius is provided in Sect. 7.1, generalising Eggleton's formula (Eggleton 1983) to situations where a radiation-pressure force is present. The necessity to abandon the Roche-lobe concept in the case of stars suffering from radiatively- or pulsation-driven wind mass loss is demonstrated in Sect. 7.4. Conclusions are drawn in Sect. 8.

\section{The different modes of wind mass loss}

Holzer \& MacGregor (1985), Schatzman et al. (1993), Lamers (1997), Willson (2000) and Owocki (2004) have reviewed the 
different types of winds existing across the Hertzsprung-Russell diagram according to their respective driving mechanisms. These mechanisms may be grouped in three broad classes: radiation-driven winds (associated with high-luminosity objects), pulsation-initiated winds, and Alfvén wave-induced winds.

The first class includes line-driven winds, operating in $l u$ minous hot stars (OB stars, and Wolf-Rayet - WR - stars as well) on resonance and subordinate lines (Castor et al. 1975; Abbott 1982). Fast winds with terminal velocities of the order of 500-3000 km s${ }^{-1}$ are generated (Kudritzki \& Puls 2000). At the same luminosities as O stars, WR stars have higher mass-loss rates, so another mechanism - like pulsation-driven mass loss, or multiple scattering of photons in an optically-thick wind with an ionisation stratification - is probably adding to radiation pressure on atomic lines (Glatzel et al. 1993; Owocki \& Gayley 1999; Nugis \& Lamers 2000; Owocki 2004). Dust-driven winds belong to the same category as radiation-driven winds, but operate instead in luminous cool stars on the asymptotic giant branch and require wind densities high enough to couple dust with gas (Gail \& Sedlmayr 1987; Lamers 1997; Wachter et al. 2002; Schröder et al. 2003; Sandin \& Höfner 2003; Sandin 2008). Wind terminal velocities are small, of the order of $10-15 \mathrm{~km} \mathrm{~s}^{-1}$. An important specificity of cool-star winds is that the driving force only slightly exceeds the gravitational attraction, as apparent from their low terminal velocities.

The dust formation requires another process to lift the matter high enough above the photosphere in the region where the temperature is less than $1500 \mathrm{~K}$. This can be done through shock waves associated with stellar pulsation (Bowen 1988), like in Mira or semi-regular variables.

Alfvén waves are the other main class of waves driving winds in stars with open magnetic field lines. This wind mechanism is important for stars that are not luminous enough to have a strong radiation pressure, i.e., magnetic $A, F, G, K$ and $M$ stars with luminosities lower than about $50 L_{\odot}$ (see Sect. 5). It is the leading candidate to account for the solar wind. This driving process has the important property of being a local phenomenon which is not derived from a potential, unlike radiation pressure.

\section{The effective potential}

In the Roche model, the two components of a binary system are considered as point sources in circular orbits and in synchronous rotation with the orbital motion. It is then possible to define a reference frame in uniform rotation about the centre of mass of the system in which the two stars are at rest. When distances are expressed in units of the orbital separation, time in units of the orbital period and masses in units of the total mass $\left(M_{1}+M_{2}\right)$, the effective potential of the system, including the gravitational and the centrifugal potentials, is given by

$\Phi=-\frac{\mu}{r_{1}}-\frac{1-\mu}{r_{2}}-\frac{x^{2}+y^{2}}{2}$

where

$\mu=\frac{M_{1}}{M_{1}+M_{2}}$

and

$r_{1}=\left((x+1-\mu)^{2}+y^{2}+z^{2}\right)^{1 / 2}$

$r_{2}=\left((x-\mu)^{2}+y^{2}+z^{2}\right)^{1 / 2}$ are respectively the distance of a test particle located at $(x, y, z)$ to the primary star (labelled " 1 " and located at $x_{1}=\mu-1$ ) and to the companion (labelled " 2 " and located at $x_{2}=\mu$ ). The centre of mass is located at the origin of the coordinate system. The mass of the test particle is supposed to be small enough not to disturb the potential. When additional forces derived from a potential are present in the system (other than the gravitational and centrifugal ones), the effective potential is

$\Phi=\Phi_{\text {extra }}-\frac{\mu}{r_{1}}-\frac{1-\mu}{r_{2}}-\frac{x^{2}+y^{2}}{2}$.

When the extra force is caused by radiation pressure, $\Phi_{\text {extra }}$ is easy to evaluate because the radiation force has the same $1 / r^{2}$ dependence on the distance as the gravitational attraction and it also pervades all space. The validity of this simple model is further discussed in Sect. 4. The ratio $f$ of the radiation to the gravitational force of star 1

$f \equiv-\frac{1}{\rho} \frac{\mathrm{d} P_{\mathrm{rad}}}{\mathrm{d} r} r_{1}\left(\frac{G M_{1}}{r_{1}^{2}}\right)^{-1}$

is independent of $r_{1}$ provided that the radiation flux at position $r_{1}$ emanating from star 1 - denoted $F_{v, 1}\left(r_{1}\right)$ - and appearing in the expression

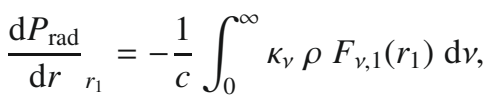

may be replaced by $L_{v, 1} /\left(4 \pi r_{1}^{2}\right)$, so that

$f=\frac{1}{4 \pi c G M_{1}} \int_{0}^{\infty} \kappa_{v} L_{v, 1} \mathrm{~d} v$.

In the above equations, $\rho$ is the density of the circumstellar matter, $\kappa_{v}$ is the absorption coefficient per unit mass at frequency $v, c$ the speed of light, and $L_{v, 1}$ the luminosity of star 1 in the frequency range $(v, v+\mathrm{d} v)$. We will ignore radiation shielding and assume that the medium is optically thin (see also Huang \& Taam 1990; Drechsel et al. 1995). If radiation does not reach thermal equilibrium with circumstellar matter (i.e., the matter is optically thin), the radiation luminosity remains approximately constant all over, and the radiation flux can be expressed as $F_{v, 1}\left(r_{1}\right)=L_{v, 1} / 4 \pi r_{1}^{2}$, which may be rewritten $F_{v, 1}\left(r_{1}\right)=F_{v, 1}^{*}\left(R_{1} / r_{1}\right)^{2}, F_{v, 1}^{*}$ being the radiation flux emitted per unit surface by the star of radius $R_{1}$. If that star is tidally distorted, limb- and gravity-darkened, a rigourous treatment would imply that Eq. (3) depends on the spherical coordinates $\theta, \phi$ since the flux irradiated by star 1 in a given direction defined by $\theta, \phi$ will now depend on these variables. The inclusion of these complications is beyond the scope of this paper, but they are not expected to alter our general conclusions. It is assumed as well that $\kappa_{v}$ does not depend strongly on the optical depth $\tau$ in the wind, i.e. that the ionisation state and/or level population of the gas do not change. This issue has been thoroughly studied in the context of radiatively-driven hot-star winds by Abbott (1982), Pauldrach et al. (1986), Shimada et al. (1994), Gayley (1995), Lamers (1997) and Puls et al. (2000). We thus consider here an idealised situation where $f$ is assumed constant. Under these hypotheses, the effective gravity of the mass-losing star is then reduced by a factor $(1-f)$, the same everywhere, and the effective potential becomes

$\Phi=-\frac{\mu(1-f)}{r_{1}}-\frac{1-\mu}{r_{2}}-\frac{x^{2}+y^{2}}{2}$ 
However, even though the equipotentials give an idea of the flow geometry, its precise description is only given by the equation of motion:

$\frac{\mathrm{d}^{2} \boldsymbol{r}}{\mathrm{d} t^{2}}=-\frac{1}{\rho} \nabla P-2 \boldsymbol{\Omega} \times \frac{\mathrm{d} \boldsymbol{r}}{\mathrm{d} t}-\nabla \Phi+\boldsymbol{F}^{\prime}$,

which includes the important Coriolis term. In the above expression, $P$ is the gas pressure (this term can be important in the case of very dense winds like in Wolf-Rayet stars), $\boldsymbol{\Omega}$ is the angular velocity, $\boldsymbol{F}^{\prime}$ is the extra force and $\Phi$ is the Roche potential (Eq. (1)). This equation of motion will not be used in the present paper.

\section{How to correctly account for radiation pressure}

The impact of radiation pressure on the shape of the equipotential surfaces has been discussed by several authors (Schuerman 1972; Huang \& Taam 1990; Drechsel et al. 1995; Howarth 1997; Maeder \& Meynet 2000; Howarth \& Smith 2001; Phillips \& Podsiadlowski 2002; Owocki 2007), in slightly different contexts, and there has been some controversy as to whether or not this effect modifies the Roche geometry. To make the discussion clear, one should distinguish three different situations: (i) the effect of radiation pressure on the matter outside the stars and flowing in the binary system; (ii) the impact of the external radiation field (from the companion star) on the equilibrium shape of the irradiated star; and (iii) the effect of the star's own radiation pressure on its equilibrium configuration.

The first effect is relevant in deriving the flow geometry, and the radiation pressure must always be included in the computation. Complications here are only related to possible shielding effects. In the present paper, we consider the situation where radiation pressure comes only from the primary and neglect any shielding by the companion star or by the circumstellar material which remains optically thin everywhere. Case (ii) has been considered by Drechsel et al. (1995), Phillips \& Podsiadlowski (2002) and Owocki (2007) in detail. In the present approach, it is not accounted for and would require the incorporation of a new $\left(1-f^{\prime}\right)$ factor reducing the gravity of the star labelled 2 in Eq. (5). Drechsel et al. (1995) and Phillips \& Podsiadlowski (2002) have performed detailed numerical calculations for this situation, including geometrical shielding effects (not considered in our simple analytical approach) but the impact is usually small. Case (iii) is conceptually more intricate and over the years has become controversial (Friend \& Castor 1982; Howarth 1997; Maeder \& Meynet 2000; Howarth \& Smith 2001). To understand why, one should first distinguish the optically-thick from the optically-thin regimes, following the insightful discussion of Friend \& Castor (1982). In optically-thick regions, the diffusion approximation for the radiative transfer may be used, resulting in an isotropic pressure with the (thermal equilibrium) value $(1 / 3) a T^{4}$. Therefore, in the hydrostatic layers below the stellar photosphere where this regime holds (this excludes WR stars), the effect of radiation should be included in the pressure term in the hydrostatic equilibrium equation and the gravitational potential is unmodified. On the other hand, in an optically-thin region, the radiation field is partially decoupled from the gas and the stellar flux can be treated in a free-streaming approximation. In this regime, the radiative flux is radial and proportional to $1 / r^{2}$ and acts as a repulsive force that reduces the gravitational force by a factor $(1-f)$.

The diffusion and streaming approximations hold respectively deep within the star and in the outer layers of wind.

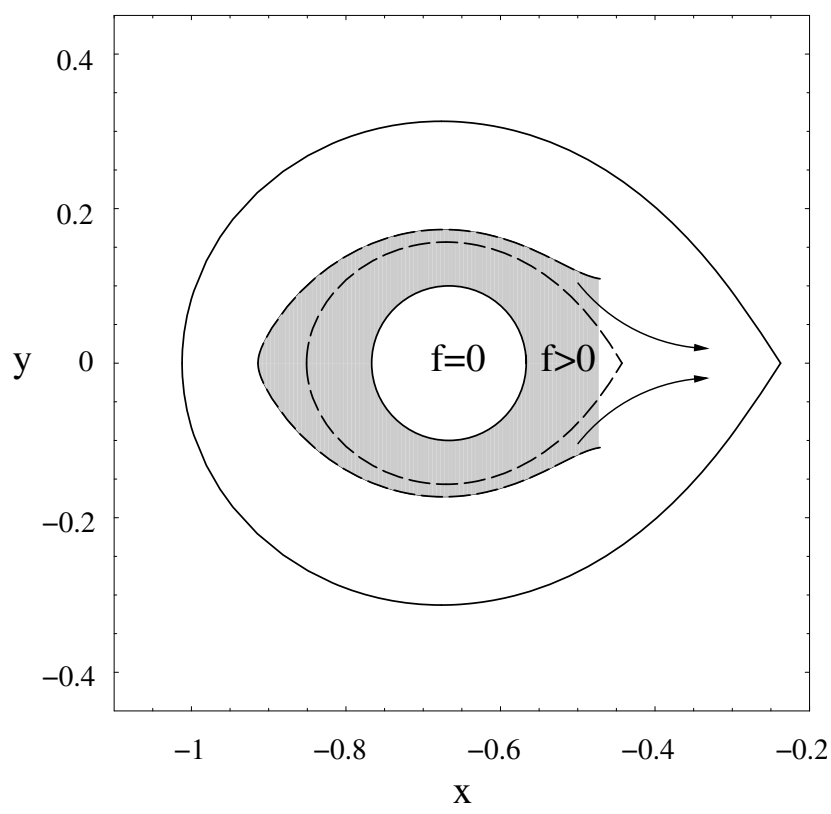

Fig. 1. A giant star with an extended atmosphere (grey region, on an exaggerated scale). As discussed in the text, in the optically-thin $(\tau<2 / 3)$ layers of the atmosphere, the ratio $f$ of the radiative force to gravitational attraction is positive and affects the Roche potential (dashed lines). Within the photosphere, the matter is optically thick, so that $f=0$ and the classical Roche potential applies (solid lines). The part of the atmosphere located above its critical Roche lobe will flow through the companion (depicted by the arrows).

Near the photosphere or in the surface layers where the hydrostatic equilibrium condition breaks down (like in WR stars, see Sect. 7.4) the situation is more ambiguous as von Zeipel's theorem does not apply (Howarth 1997).

To illustrate this dichotomy, consider Fig. 1 which shows the Roche-lobe filling criterion in a giant star at $(f=0)$ and above $(f>0)$ the photosphere where the radiation pressure modifies the potential. The unmodified Roche potential (Eq. (1)) should be used for the subphotospheric matter, whereas the modified potential (Eq. (5)) should be used above the photosphere (grey area on Fig. 1). Since the modified potential leads to a smaller critical surface (as will be shown in Sect. 7.1), the situation may arise where the photosphere does not fill its Roche lobe, but the supra-photospheric matter does, especially for (super) giant stars with extended atmospheres. These supra-photospheric shells are removed from the star, and in the case of a giant star with a convective envelope, this removal will cause the photosphere to expand on a thermal time scale (Ritter 1996). Therefore, even though the photosphere itself does not fill its own Roche lobe, the consideration of the modified Roche potential alters the evolution, at least for giant stars. This distinction between dwarfs and giants is important, because empirical arguments call for the use of a modified Roche equipotential for giants, but not for dwarfs. Howarth (1997) has given convincing arguments that the unmodified potential should be used whenever there is evidence for gravity darkening (a direct consequence of von Zeipel's theorem), as is the case for dwarf stars in binary systems (Anderson \& Shu 1977; Rafert \& Twigg 1980). The situation is however different for giants: an important empirical motivation for considering the case $f>0$ is that it may account for the fact that some giant stars exhibit ellipsoidal variations (due to non-sphericity) despite small (classical) Roche-filling factors. Those ellipsoidal variables would appear enigmatic without the 
presence of an additional force reducing the actual Roche radius and disturbing the stellar shape. As it will be shown in Sect. 7.1, the modified Roche radius (for $f>0$ ) is smaller than the classical one (for $f=0$ ). Therefore, stars nearly filling their Roche lobe with $f>0$ would be far from filling their lobe if that filling factor were estimated with the traditional formula corresponding to $f=0$. This is probably what happens for the 13 s-type symbiotic systems with ellipsoidal variations detected by Mikołajewska (2007), which have a (classical) Roche filling factor of only 0.4-0.5. Based on the analysis of the orbital circularisation in a sample of binary systems with M-giant primaries, Frankowski et al. (2009) find that these giants also do not fill more than $\sim 0.5$ of their classical Roche lobes. Again, the explanation may lie in a decrease in the Roche radius below its classical value.

\section{Typical $f$ values}

We now evaluate the $f$ factor using the Castor-Abbott-Klein (Castor et al. 1975, hereafter CAK) theory of line-driven winds. Let $g_{\text {grav }}(r)=\frac{G M}{r^{2}}$ be the gravitational acceleration at distance $r$ from the stellar centre, and

$g_{\mathrm{rad}} \equiv g_{\mathrm{Th}}(1+\mathcal{F}(\tau))=\frac{\kappa_{\mathrm{e}} L}{4 \pi c r^{2}}(1+\mathcal{F}(\tau))$,

be the sum of the radiative accelerations due to Thomson scattering $\left(g_{\mathrm{Th}}\right)$ and to an ensemble of lines $\left(g_{\mathrm{Th}} \mathcal{F}(\tau)\right)$, where $\mathcal{F}$ is the so-called "force multiplier". In the above relation, $\kappa_{\mathrm{e}}=\sigma_{\mathrm{e}} n_{\mathrm{e}} / \rho$ is the opacity coefficient per unit mass for Thomson scattering, $\sigma_{\mathrm{e}}$ is the corresponding Thomson cross section $(6.65 \times$ $\left.10^{-25} \mathrm{~cm}^{-2}\right), n_{\mathrm{e}}$ is the electron number density and $\rho$ is the density of the wind. If the wind is fully ionised, $\kappa_{\mathrm{e}}=\sigma_{\mathrm{e}} \frac{1}{m_{\mathrm{H}}}\left\langle\frac{Z}{A}\right\rangle \sim$ $0.2(1+X) \mathrm{cm}^{2} \mathrm{~g}^{-1}$, where $m_{\mathrm{H}}$ is the hydrogen mass. The force multiplier has been computed by CAK, Abbott (1982), Pauldrach et al. (1986), Shimada et al. (1994), Gayley (1995), Lamers (1997) and Puls et al. (2000) for various wind thermodynamical conditions. It turns out to be very small at optical depths close to one because of the "saturation effect": radiation with frequencies matching those of atomic transitions are efficiently removed from the flux at the base of the photosphere and no flux at those frequencies is left further up in a static atmosphere to accelerate matter. Of course, the situation changes in the presence of a wind, since the associated velocity gradient will then induce a Doppler shift of the line frequencies. At low optical depths (i.e., higher up in the expanding atmosphere), when all lines become optically thin, $\mathcal{F}(\tau)$ becomes very large, and reaches asymptotic values up to about 2000 (see e.g., Table 2 of Abbott 1982, also Gayley 1995).

Using the Eddington parameter

$\Gamma=\frac{\kappa_{\mathrm{e}} L}{4 \pi c G M}$

we find that

$f=\Gamma(1+\mathcal{F})$.

In Table 1 , we have listed the $f$ and $\Gamma$ values for stars along the main sequence. The numbers are obtained by considering an optically-thin medium, where $\mathcal{F}$ reaches its maximum value of about 2000 (Abbott 1982; Gayley 1995).

The threshold $f=1$ is found around spectral type A0 (corresponding to stars with luminosities less than about $50 L_{\odot}$ ), and this result is consistent with empirical arguments stating that the
Table 1. The Eddington parameter $\Gamma$ (Eq. (8)) for stars along the main sequence, adopting $\kappa_{\mathrm{e}}=0.35 \mathrm{~cm}^{2} \mathrm{~g}^{-1}$. Since $\mathcal{F}<2000$ (Abbott 1982), $f_{\max }=2000 \Gamma$.

\begin{tabular}{lllll}
\hline \hline Sp. Typ. & $\log \left(L / L_{\odot}\right)$ & $\begin{array}{l}M \\
\left(M_{\odot}\right)\end{array}$ & $\Gamma$ & $f_{\max }$ \\
\hline O5V & 5.95 & 60.0 & $4.0 \times 10^{-1}$ & 807. \\
B0V & 4.77 & 17.5 & $9.2 \times 10^{-2}$ & 184. \\
B5V & 2.97 & 5.9 & $4.3 \times 10^{-3}$ & 8.68 \\
B8V & 2.33 & 3.8 & $1.5 \times 10^{-3}$ & 3.06 \\
A0V & 1.77 & 2.9 & $5.5 \times 10^{-4}$ & 1.10 \\
A5V & 1.19 & 2.0 & $2.1 \times 10^{-4}$ & 0.42 \\
F0V & 0.86 & 1.6 & $1.3 \times 10^{-4}$ & 0.25 \\
F5V & 0.51 & 1.4 & $6.4 \times 10^{-5}$ & 0.13 \\
\hline
\end{tabular}

Luminosities and masses are from Cox (2000).

Table 2. The $f$ parameter for $M=1 M_{\odot} \mathrm{K}$ and $\mathrm{M}$ giants and subgiants with $\mathrm{C} / \mathrm{O}=0.5$, at $\tau_{\text {Ross }}=2 / 3$.

\begin{tabular}{ccc}
\hline \hline$T_{\text {eff }}(\mathrm{K})$ & $\log g$ & $f$ \\
\hline 5000 & 0 & 0.97 \\
4000 & 0 & 0.43 \\
3000 & 0 & 0.18 \\
5000 & 3 & $9.6 \times 10^{-4}$ \\
4000 & 3 & $4.3 \times 10^{-4}$ \\
3000 & 3 & $1.2 \times 10^{-4}$ \\
\hline
\end{tabular}

peculiarities of Am stars cannot survive if the mass loss is too strong (Michaud et al. 1983; Michaud \& Charland 1986; Lemke 1990; Babel 1992). Furthermore, the very existence of Am stars demonstrates the importance of the radiative acceleration on the distribution of the various chemical elements of these stars (e.g. Hui-Bon-Hoa et al. 2001; Alecian \& LeBlanc 2002).

For late-type stars, the CAK model cannot be used because this model does not include the relevant transitions, especially molecular lines which may contribute significantly to the radiative driving force (Jorgensen \& Johnson 1992). Therefore, radiative accelerations for late-type stars have been taken from the MARCS model atmospheres (Gustafsson et al. 2008) for K and $\mathrm{M}$ giants. For these stars, the radiation pressure is dominated by the contribution of the near-infrared continuum where they emit most of their radiation. The corresponding $f$ values are listed in Table 2.

For AGB stars with still higher luminosities than those considered in Table 2, the radiation driving force is now dominated by absorption and scattering in molecular lines, yielding values of $f$ as large as 0.15 for $\mathrm{C}$-type stars with $\mathrm{C} / \mathrm{O}=2, \log g=-1$ and $T_{\text {eff }}=2500 \mathrm{~K}$ (Elitzur et al. 1989; Jorgensen \& Johnson 1992).

In the next sections, the values of $f$ quoted above will be applied to different astrophysical situations. It has to be made very clear that one should distinguish situations involving RLOF (Sect. 7) from situations involving modifications of the geometry of the equipotential surfaces far above the photosphere of the mass-losing star (Sect. 6). Although in the latter case, the $f$ values provided in Table 1 may be used without restriction, the situation is more complicated in the former case. This is because the RLOF criterion involves photospheric layers, and we have argued in Sect. 4 that non-zero $f$ values associated with radiation pressure do not usually alter the stellar equilibrium configuration (according to the von Zeipel theorem), except in special circumstances involving giant stars. The two situations are therefore discussed in separate sections below. 


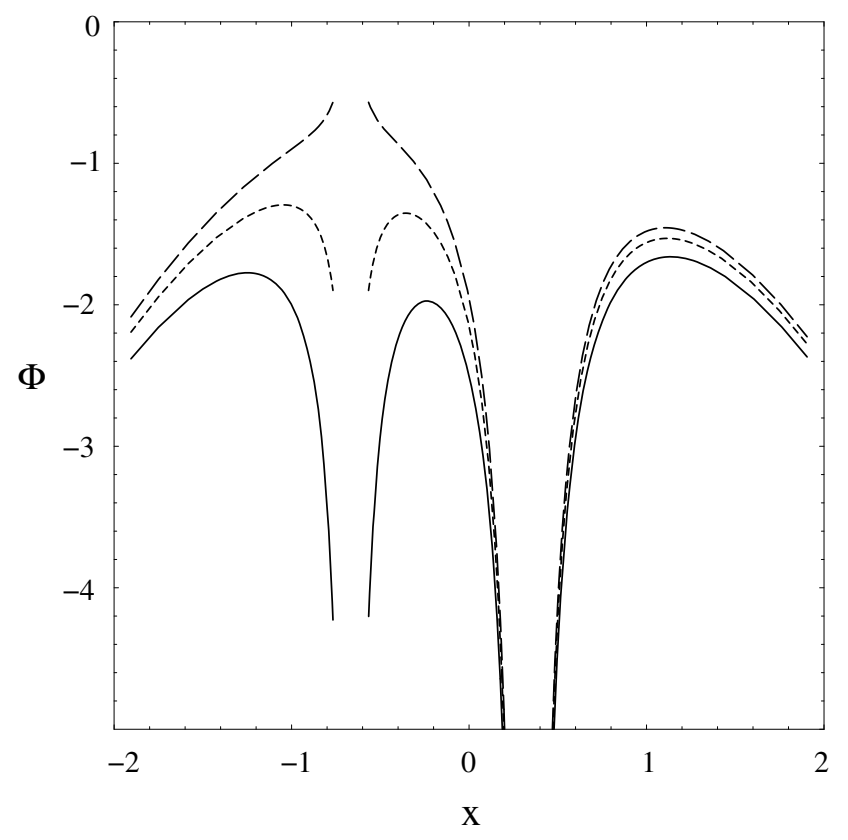

Fig. 2. Cross-sections through the effective equipotential surfaces (Eq. (5)) along the line joining the two stars, for $\mu=1 / 3$. The masslosing star (located at $x_{1}=-2 / 3$ ) is the less massive component and has a (dimensionless) radius of 0.1 . Three cases are depicted: $f=0$ (solid line); $f=0.7$ (short-dashed line; note that the Roche lobe is decreasing compared to the case $f=0$ ); $f=1.1$ (long-dashed line: there is no Roche lobe any longer).

\section{The modified Roche equipotentials}

Figure 2 presents sections through the effective equipotential surfaces (Eq. (5)) along the line joining the two stars, for $\mu=$ $1 / 3$, i.e. the mass-losing star (located at $x_{1}=-2 / 3$ ) is the less massive component, and for three different values of $f: f=0$ (solid line), $f=0.7$ (short-dashed line), and $f=1.1$ (longdashed line). The first case corresponds to a situation with no extra force, the second to a situation where the extra force is present but not sufficient to drive mass loss (note that the Roche lobe is becoming smaller as compared to the case $f=0$ ), and the third case corresponds to a situation where the extra-force is actually driving the mass loss. Note that in this case, there is no longer a Roche lobe.

Figure 3 presents the different families of equipotentials as a function of $f$ and $\mu$, when $f<1$. Panels $\mathrm{b}$ and $\mathrm{c}$ correspond to the critical configurations where the Lagrangian points $L_{2}$ and $L_{3}$ or $L_{1}$ and $L_{2}$ are located on the same equipotentials. These situations are encountered for the specific values of $f$ denoted $f_{1}(\mu)$ and $f_{2}(\mu)$, respectively. These functions are approximated by the following expressions with a relative error smaller than $1 \%$ for $f_{1}(\mu)$ and $2 \%$ (when $\mu \leq 0.97$ ) for $f_{2}(\mu)$ :

$$
\begin{aligned}
& f_{1}(\mu)=(1-2 \mu)\left(1-0.80 \mu^{1 / 2}+0.82 \mu\right) \\
& f_{2}(\mu)=(1-\mu)\left(1-0.72 \mu^{1 / 2}+0.66 \mu^{3 / 2}+\mu^{17}\right) .
\end{aligned}
$$

The term $\mu^{17}$ is necessary to obtain a good fit to $f_{2}(\mu)$ when $\mu$ approaches 1 .

The different regions delineated by $f_{1}$ and $f_{2}$ in Fig. 4 correspond to different equipotential topologies. Systems with $f<f_{1}$, $f=f_{1}, f_{1}<f \leq f_{2}$ and $f>f_{2}$ are topologically similar to cases displayed in Fig. 3 in panels a, b, c and d, respectively. In the last case (panel d), the Roche lobes of the two components do not belong to the same equipotential. In contrast to the standard case, the matter ejected by the primary is not necessarily transferred directly into the Roche lobe of the companion, but all or a fraction of it may instead feed a circumbinary disc. As mentioned in Sect. 1, this issue is important as such discs are very common in binaries involving low- and intermediate-mass components. The Coriolis force, which is not conservative, also plays a major role in the formation of such discs, as shown by numerical simulations (Theuns \& Jorissen 1993; Mastrodemos \& Morris 1998, 1999; Sytov et al. 2009).

\section{The RLOF criterion}

\subsection{The modified Roche radius}

A generalisation to the cases $0 \leq f \leq 1$ of the Eggleton (1983) formula for the radius of a sphere with the same volume as the Roche lobe, is given by

$\mathcal{R}(q, f) \equiv \frac{R_{\mathrm{R}}(q, f)}{a}=\frac{A(f) q^{2 / 3}}{B(f) q^{2 / 3}+\ln \left(1+C(f) q^{1 / 3}\right)}$

where

$A(f)=(1-f)^{1 / 3}\left(0.49+0.25 f+0.35 f^{2}-0.59 f^{3}+0.37 f^{4}\right)$,

$B(f)=0.6+0.3 f$,

$C(f)=1+f$,

$q \equiv M_{1} / M_{2}$ is the mass ratio of the two components (star 1 being the one with non-negligible radiation pressure) and $a$ is the orbital separation. For $f=0$, the Eggleton (1983) expression is recovered. Figure 5 shows the Roche radius as a function of the mass ratio $q$ for the values $f=0, f=0.5$ and $f=0.9$. The Roche radius is clearly smaller in the presence of radiation pressure and is reduced by a factor of $\sim 2$ between the cases $f=0$ and $f=0.9$. The Roche lobe vanishes (and its radius thus goes to zero) when the radiation force becomes equal to the gravitational attraction (i.e. $f=1$ ).

The analytical fit to the Roche radius as approximated by Eq. (12) is accurate to better than $3 \%$ over the extended range $0.1 \leq q \leq \infty$ and $0 \leq f \leq 0.95$. Outside this parameter range, the relative error is less than $7 \%$. The fit is based on numerical results obtained with the method outlined by Huang \& Taam (1990). We emphasize once again that, according to the discussion of Sect. 4, the Roche radius expressed by Eq. (12) applies only to stars with an extended atmosphere where the freestreaming approximation holds for the radiation field.

\subsection{RLOF stability}

We now evaluate the impact of the above modifications on the RLOF stability. In the Roche model, the stability condition imposes that, when the star fills its Roche lobe, subsequent mass loss does not lead to a runaway situation. It is expressed by the condition $\zeta_{\mathrm{R}}<\zeta$, where

$\zeta_{\mathrm{R}} \equiv \frac{\mathrm{d} \ln R_{\mathrm{R}}}{\mathrm{d} \ln M_{1}}$

and

$\zeta \equiv \frac{\mathrm{d} \ln R_{1}}{\mathrm{~d} \ln M_{1}}$

are the Roche-lobe mass-radius exponent and the mass-radius exponent of the donor, respectively. A star responds to mass loss on two timescales. The immediate response is on the adiabatic time scale $\left(\tau_{\mathrm{dyn}}\right)$, after which hydrostatic equilibrium is restored 

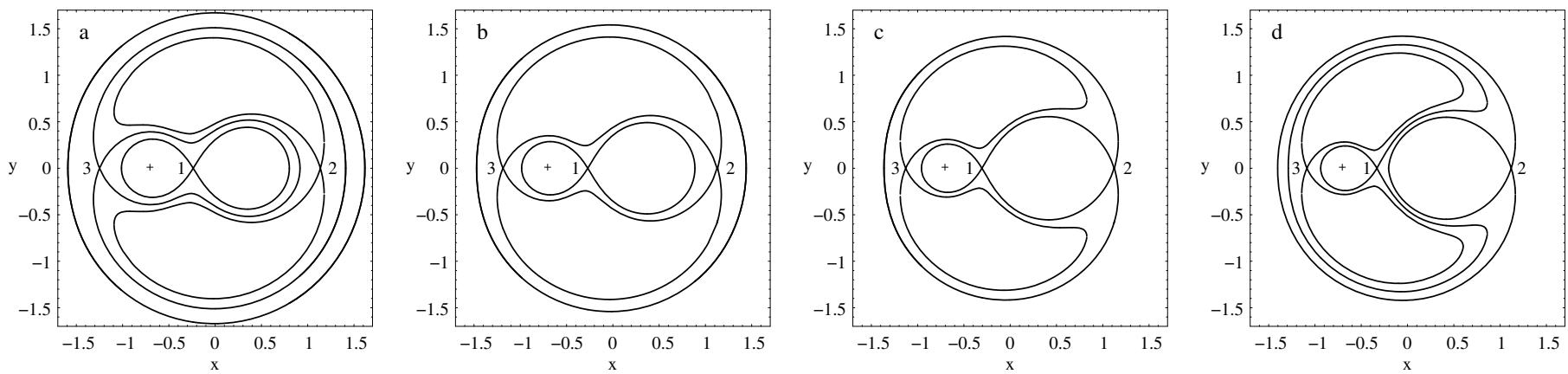

Fig. 3. Section in the orbital plane of the Roche equipotentials for a binary system with a dimensionless mass $\mu=1 / 3$ and $f=0$ (panel a)), the critical values $f_{1}=0.27$ (panel b)) and $f_{2}=0.48$ (panel c)), obtained from numerical integration. The case $f=0.6$ is presented in panel d). The primary star, located at $x=\mu-1$, is depicted by the cross. The Lagrangian points are labelled 1,2 and 3 .

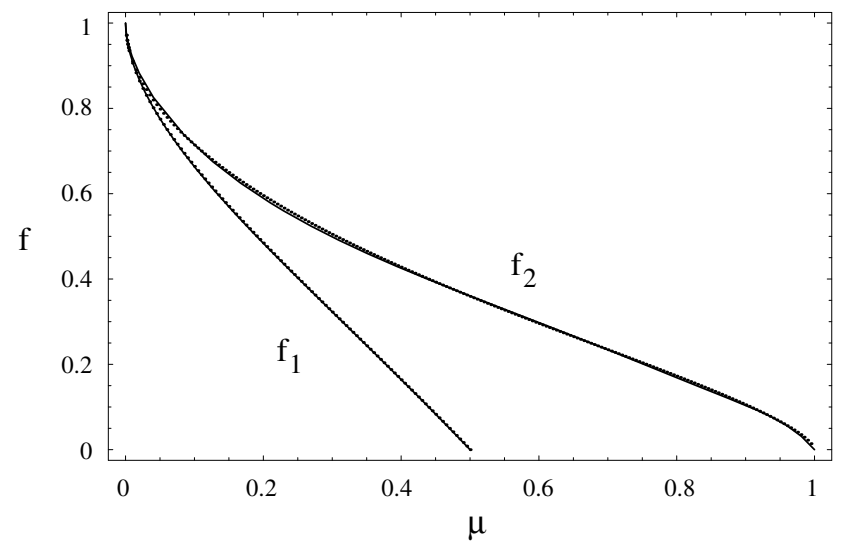

Fig. 4. The functions $f_{1}(\mu)$ and $f_{2}(\mu)$ (see text), obtained by numerical integration (dots) and their analytical fit (solid curves) as expressed by Eqs. (10) and (11). The three regions delineated by the curves in the $(f, \mu)$ plane correspond to three different topological structures for the equipotentials (see text and Fig. 3).

but negligible heat transport has occurred. The mass-radius exponent characterizing this adiabatic readjustment is denoted by $\zeta_{\text {ad }}$. On the other hand, thermal equilibrium is recovered on the Kelvin-Helmoltz time scale $\left(\tau_{\mathrm{KH}}\right)$ and is characterised by the exponent $\zeta_{\text {th }}$. The adiabatic and thermal stability conditions become respectively

$\zeta_{\mathrm{R}}<\zeta_{\mathrm{ad}}$

and

$\zeta_{\mathrm{R}}<\zeta_{\text {th }}$.

If neither of these conditions is satisfied, mass transfer proceeds on the fastest of the two timescales.

To estimate $\zeta_{\mathrm{R}}$, Soberman et al. (1997) (see also Jorissen 2003) assume that a fraction $\alpha$ of the mass lost in the wind escapes to infinity, that a fraction $\eta$ is accreted by the companion and the remaining $\delta=1-\alpha-\eta$ goes into feeding a circumbinary disc of radius $a_{r}=\gamma^{2} a$. In this framework the Roche-lobe mass-radius exponent is

$$
\begin{aligned}
& \zeta_{\mathrm{R}}=2(\left.\frac{\alpha}{1+q}+\gamma \delta(1+q)-\lambda\right)+\frac{q}{1+q}(1-\eta) \\
&+2(\eta q-1)+\frac{\mathrm{d} \ln \mathcal{R}}{\mathrm{d} \ln q}(1+\eta q),
\end{aligned}
$$

where $\lambda \sim 0.05$ according to the hydrodynamical simulations of Theuns et al. (1996).

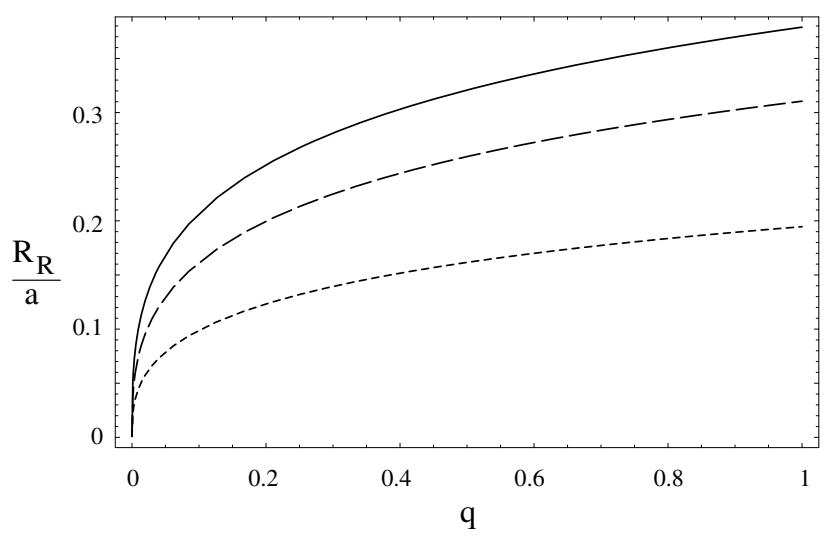

Fig. 5. The normalised Roche radius $R_{\mathrm{R}} / a$ (where $a$ is the orbital separation) as a function of the mass ratio $\left(q \equiv M_{1} / M_{2}\right)$ for the cases $f=0$ (solid line), $f=0.5$ (long-dashed line) and $f=0.9$ (short-dashed line).

When an extra force is present, the term $(\mathrm{d} \ln \mathcal{R} / \mathrm{d} \ln q)$ increases and may possibly destabilise the system. However, the effect is small $(<10 \%$ for $f$ ranging between 0 and 0.9$)$ and the stability condition remains always dominated by the first three terms of Eq. (17), which depend on the efficiency of mass transfer through the parameters $\eta, \delta$ and $\gamma$. This is apparent from Fig. 4 of Soberman et al. (1997) or Fig. 9.14 of Jorissen (2003). The direct effect of the extra force on RLOF stability is consequently negligible.

\subsection{Critical period}

An interesting application of Eq. (12) is related to the critical period $P_{\text {crit }}$, the orbital period below which RLOF occurs, given by (Eggleton 2006)

$$
\begin{aligned}
P_{\text {crit }} & =\left(\frac{4 \pi^{2} R^{3}}{G M}\right)^{1 / 2} \mathcal{R}(q, f)^{-3 / 2} \\
& \sim 0.1159\left(\frac{R^{3}\left(R_{\odot}\right)}{M\left(M_{\odot}\right)}\right)^{1 / 2} \mathcal{R}(q, f)^{-3 / 2} \text { (days) }
\end{aligned}
$$

where $M=M_{1}+M_{2} . P_{\text {crit }}$ increases strongly when an extra force is present as shown in Fig. 6. This means that RLOF, either stable or not, would occur at a longer orbital period than inferred from the size of the classical Roche lobe. Such an effect of the extra force (with an estimated $f=0.65-0.85$ ) shrinking the effective Roche lobe was offered by Frankowski \& Tylenda (2001) as an explanation for the symbiotic stars "avoiding" filling their 


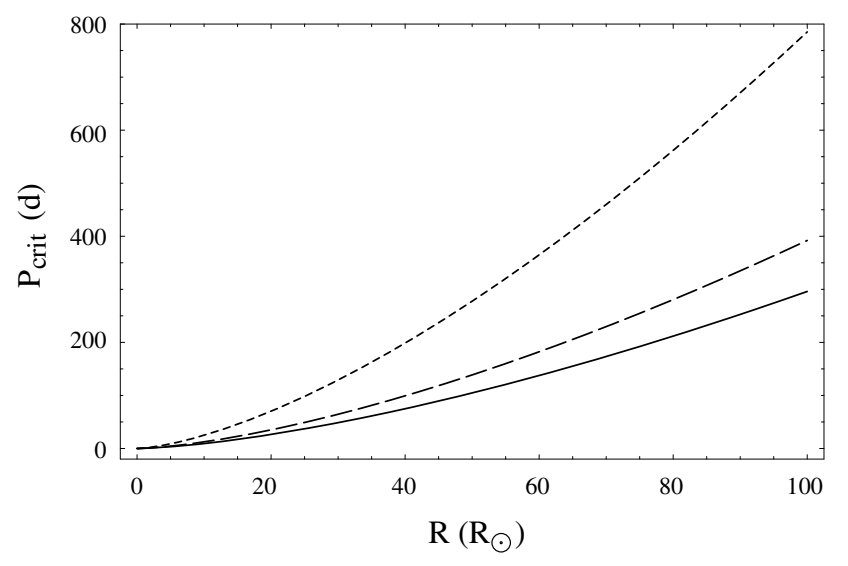

Fig. 6. Critical period $P_{\text {crit }}$ in days from Eq. (18) as a function of the stellar radius $R\left(R_{\odot}\right)$ for $f=0$ (solid line), $f=0.5$ (long-dashed line) and $f=0.9$ (short-dashed line). In each case, $M_{1}=1.2 M_{\odot}$ and $M_{2}=$ $0.6 M_{\odot}$ (i.e., $q=2$ or $\mu=2 / 3$ ).

Roche lobes (Mürset \& Schmid 1999). As noted in Sect. 4, it was also discovered that a number of symbiotics exhibit ellipsoidal variations, apparently being tidally distorted despite not quite filling their classical Roche lobes (Mikołajewska 2007). Observations of the orbital circularisation in a sample of binaries with $\mathrm{M}$ giant primaries supply another possible example of this effect: these giants do not fill more than $\sim 0.5$ of their classical Roche lobe (Frankowski et al. 2009). If this interpretation of these system properties being modified by the extra force is correct, the $f>0.7$ values implied for these cool giants are significantly higher than the estimates given for absorption/scattering in molecular lines in the photospheres of AGB stars (see Sect. 5). They are closer to the effective $f \sim 1$ values inferred from the pulsation-driven winds of AGB Mira stars. This reflects the fact that radiation pressure is not the only energy and momentum source available in the photospheres of giant stars (e.g., even non-pulsating cool giants show bulk motion, manifesting as radial-velocity jitter; Gunn \& Griffin 1979).

\subsection{No RLOF for $f>1$}

We showed in Fig. 2 that when $f>1$, there is no longer a critical Roche surface around the mass-losing star, so that the very concept of RLOF becomes meaningless.

For a star where, e.g., the radiation pressure is high enough to expel stellar material, the potential corresponds to a net repulsive force (long-dashed-line in Fig. 2) and the Roche lobe around the mass-losing star has no meaning any longer. Similarly the stellar radius needs to be re-defined, especially in the case of optically-thick winds (as for WR stars), when the photospheric radius (corresponding to an optical depth $\tau=2 / 3$ ) falls within the wind (de Loore et al. 1982; Baschek et al. 1991; Moffat \& Marchenko 1996). This property is accounted for in recent stellar models which correct the stellar radius using extrapolation of the wind expansion law in the optically-thick region (Langer 1989; Hamann 1993).

A further consequence of the absence of a Roche lobe around radiatively driven mass-losing stars is that there is no dramatic change in the mass-loss regime from wind mass loss to RLOF, as the latter is now ill-defined. In fact, several authors have already promoted this idea of a smooth transition of the mass loss rate from the wind to the RLOF regime (Tout \& Eggleton 1988; Frankowski \& Tylenda 2001).
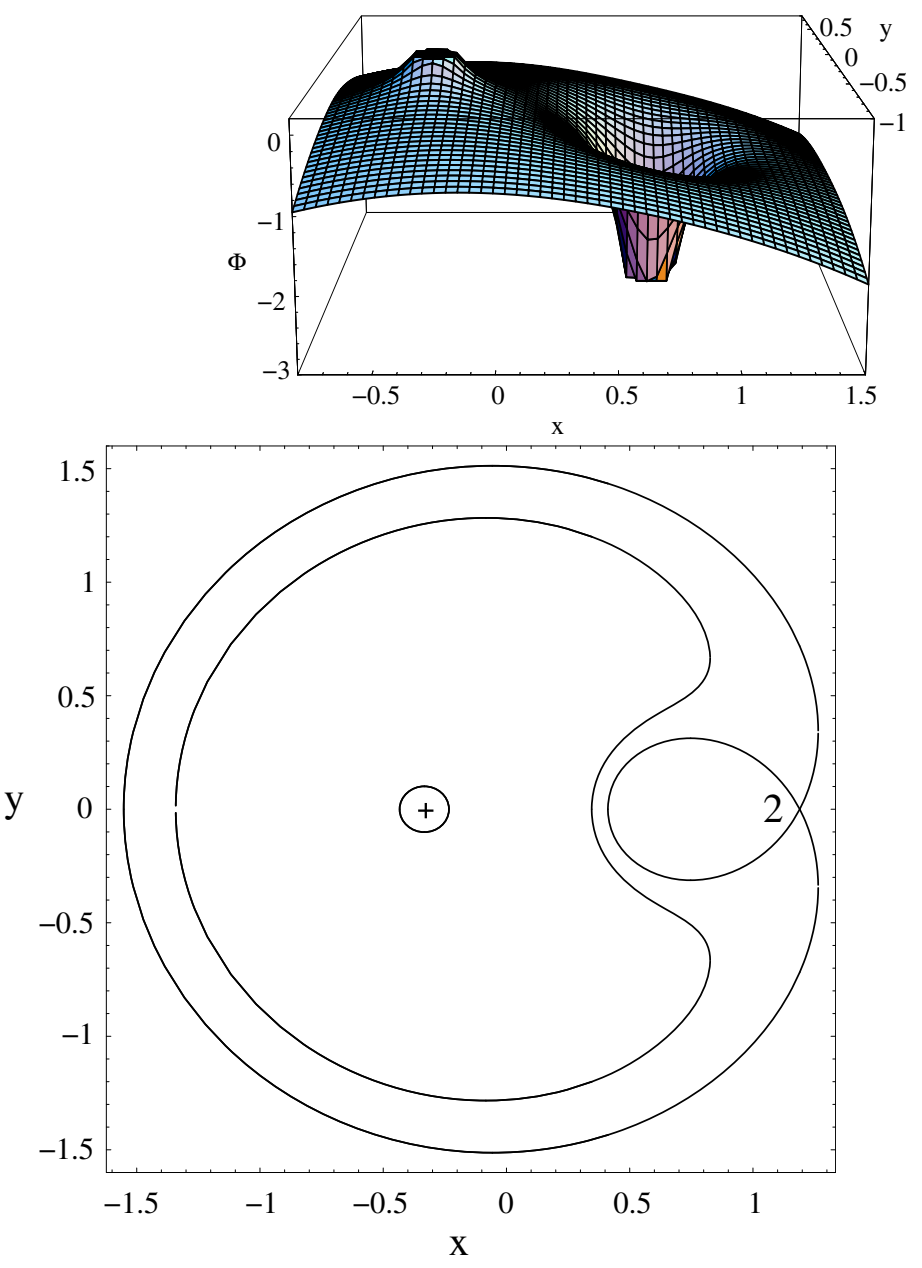

Fig. 7. Roche potential surface (upper panel) and equipotentials on the orbital plane (bottom panel) for $\mu=2 / 3$ and $f=1$.1. The mass-losing star, located at $x=\mu-1$ and depicted by the cross, has a radius of 0.1 (in units of the orbital separation). The only Lagrangian point is $L_{2}$.

An important issue related to mass transfer is to evaluate whether or not a common envelope will form and this outcome depends on the geometry of the equipotentials. Contrarily to the situation prevailing during classical RLOF, the mass lost by the wind is not necessarily injected into the Roche lobe of the companion as illustrated in Fig. 7. In particular, a substantial fraction of the wind can avoid the companion's Roche lobe and instead be used to form a circumbinary disc.

\subsection{Pulsation-driven winds: the case of Mira stars}

The process driving the wind in Mira stars seems to start with pulsation-induced shock waves that lift the matter high enough above the photosphere for dust to form. Since all photospheric particles will feel this upward force, it should be included in the effective potential. However, in the case of momentum transfer from shock waves, there is no simple mathematical expression for the extra acceleration $g_{\text {ext }}$, unlike the case of the radiationpressure force. Nevertheless, it is possible to infer the run of $g_{\mathrm{ext}}$ as a function of the distance $r_{1}$ from the stellar surface using model predictions for the wind velocity (see e.g., Bowen 1988; Willson 2000). In the steady state approximation, the acceleration $\mathrm{d} v / \mathrm{d} t$ for a wind particle is

$-g_{\mathrm{grav}}+g_{\mathrm{ext}}=\mathrm{d} v / \mathrm{d} t=v \frac{\mathrm{d} v}{\mathrm{~d} r_{1}}$. 


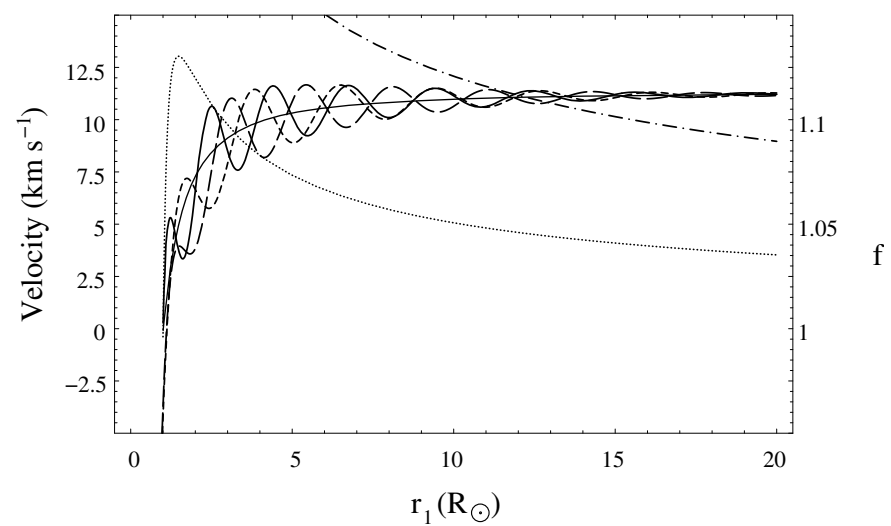

Fig. 8. Wind velocities $v_{\text {wind }}\left(r_{1}\right)$ (solid, short- and long-dashed lines and left-hand scale) and escape velocity (dot-dashed line) from model calculations of Bowen (1988) for a Mira star of $M=1.2 M_{\odot}, R=270 R_{\odot}$ and $L=5415 L_{\odot}$, including pulsation and radiation pressure on dust. The function $f\left(r_{1}\right)$ (dotted line and right-hand scale) is derived from Eq. (20).

The above relation implicitly assumes that the wind velocity function is derivable. It thus requires us to smooth the discontinuities associated with the shock waves (thick line in Fig. 8). By suppressing the non-conservative character of the shock waves, this case becomes similar to that of a conservative force deriving from a potential $\Phi_{\text {ext }}$. The fact that Mira stars have relatively low-velocity winds implies that this extra force is mostly used to lift the matter out of the potential well. The run of $f$ with distance is then written

$f\left(r_{1}\right) \equiv \frac{g_{\text {ext }}\left(r_{1}\right)}{g_{\text {grav }}\left(r_{1}\right)}=1+\frac{v}{g_{\text {grav }}\left(r_{1}\right)} \frac{\mathrm{d} v}{\mathrm{~d} r_{1}}$.

Because Mira stars are pulsating, their wind-velocity curves are time-dependent (Bowen 1988). In order to mimic this time variability, high-frequency spatial and temporal variations are added to the smooth, long-range velocity curve. The high-frequency component corresponds to a sinusoidal curve whose amplitude decreases exponentially with distance from the stellar surface. This high-frequency component is moreover phase-shifted by $\pi / 2$ (solid line in Fig. 8 ) and $\pi$ (long-dashed line) to mimick the temporal evolution (shock-wave propagation). The corresponding potentials are represented in Fig. 9 (solid, long- and short-dashed lines). Inside the stellar radius, matter is supposed to be in hydrostatic equilibrium, with gas and radiation pressure balancing gravitation, so that matter is at rest on average. At different times of the shock-wave propagation, the potential can be repulsive (solid and long-dashed curves), allowing for the ejection of matter, or becomes attractive (short-dashed line), temporarily preventing mass ejection.

In our calculations, $f$ remains on average only slightly larger than unity which confirms the fact that the driving mechanism is mainly used to work against the gravitational attraction. Finally, for binary systems involving Mira stars, the companion does influence the mass-losing star by altering the physical processes driving its wind. Frankowski \& Tylenda (2001) for instance stress that the empirical formulae fitting red-giant mass-loss rates (Reimers 1975; Arndt et al. 1997) depend on the surface gravity, and consider how gravity of the mass-losing star will be altered, both because of the straightforward addition of the companion gravitational attraction and because of the tidal distortion. Furthermore, in Mira stars, the gravitational field of the companion may also alter the source of the mass-loss, by disturbing the formation and the properties of the shock waves. All

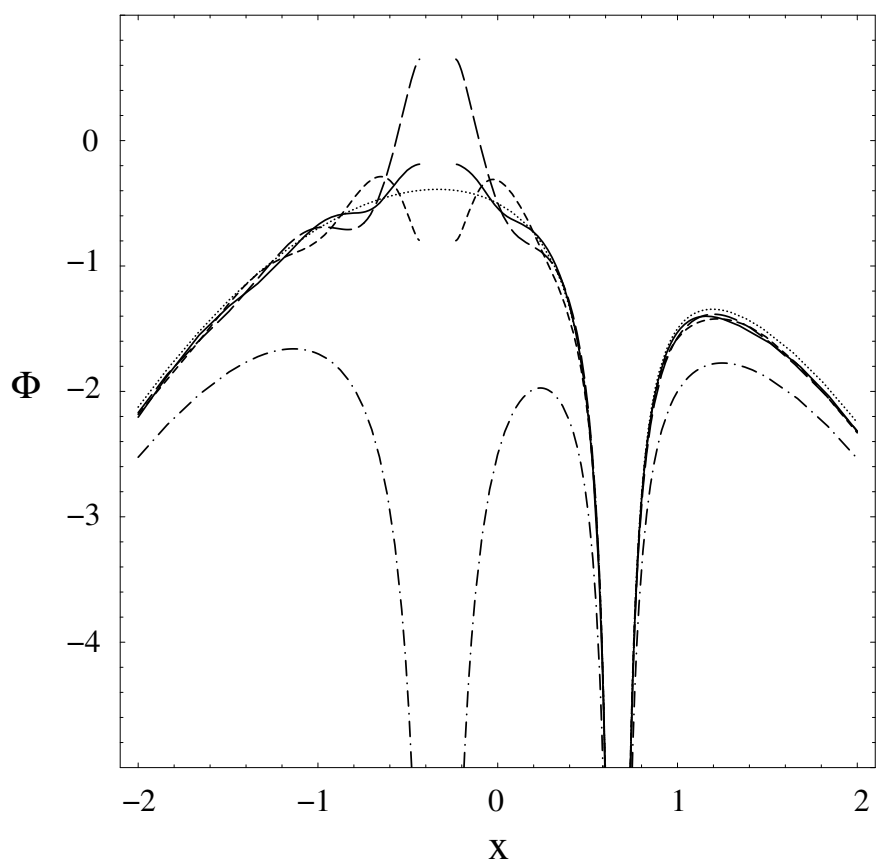

Fig. 9. Same as Fig. 2 in the case of a Mira-type wind (solid, short- and long-dashed lines), derived from the wind velocity curves displayed in Fig. 8 and using Eq. (20). The star is assumed to have a radius of 0.1 (in units of the orbital separation), and $\mu=2 / 3$. The dotted and dot-dashed lines correspond respectively to the cases $f=1$ and $f=0$.

these aspects contribute to making the determination of $f$ rather uncertain.

\section{Conclusions}

This paper presents and analyses the Roche potential modified by the presence of an extra force associated with radiation pressure or pulsation. The magnitude of this perturbing force is quantified by the parameter $f$ which represents the ratio of the extra-force and the gravitational attraction (Schuerman 1972). An estimate of this parameter for main sequence, RGB, AGB and Mira stars is also provided.

For $0<f<1$, the Roche potential may be substantially modified. In particular, if $f>f_{1}$ the deformation of the equipotentials allow the matter ejected by the mass-losing star to go into a circumbinary disc. As the extra force $(f)$ becomes stronger, the Roche radius decreases, favouring RLOF mass transfer.

Numerical fits and generalisation of the Roche radius are provided in this paper for $f<1$. It is shown that the effects of the extra force on the RLOF stability is negligible.

For $f>1$, the Roche lobe has no meaning any longer. Such situations occur in luminous stars where radiation drives the mass loss or in pulsating giant stars. In this latter case, the recurrent deposition of momentum by the shock waves in the atmosphere allows matter at the surface to be expelled.

The consideration of a modified Roche lobe is (directly or indirectly) supported by numerous observations (like the frequent occurrence of circumbinary discs in post-mass-transfer systems, and the small classical Roche-filling factors derived for symbiotic or M giants despite their ellipsoidal variability or circular orbit...) and should be taken into account.

Acknowledgements. L.S. is Research Associate from FRS-F.N.R.S., and T.D. is Boursier F.R.I.A. This work has been partly funded by an Action de recherche 
concertée $(A R C)$ from the Direction générale de l'Enseignement non obligatoire et de la Recherche scientifique - Direction de la recherche scientifique Communauté française de Belgique.

\section{References}

Abbott, D. C. 1982, ApJ, 259, 282

Alecian, G., \& LeBlanc, F. 2002, MNRAS, 332, 891

Anderson, L., \& Shu, F. H. 1977, ApJ, 214, 798

Arndt, T. U., Fleischer, A. J., \& Sedlmayr, E. 1997, A\&A, 327, 614

Babel, J. 1992, A\&A, 258, 449

Baschek, B., Scholz, M., \& Wehrse, R. 1991, A\&A, 246, 374

Bowen, G. H. 1988, ApJ, 329, 299

Castor, J. I., Abbott, D. C., \& Klein, R. I. 1975, AJ, 195, 157

Cox, A. N. 2000, Allen's Astrophysical Quantites (Athlone Press)

de Loore, C., Hellings, P., \& Lamers, H. J. G. 1982, in Wolf-Rayet Stars: Observations, Physics, Evolution, ed. C. W. H. de Loore, \& A. J. Willis, IAU Symp., 99, 53

de Ruyter, S., van Winckel, H., Maas, T., et al. 2006, A\&A, 448, 641

Djurasevic, G. 1986, Ap\&SS, 124, 5

Drechsel, H., Haas, S., Lorenz, R., \& Gayler, S. 1995, A\&A, 294, 723

Eggleton, P. P. 1983, ApJ, 268, 368

Eggleton, P. 2006, Evolutionary Processes in Binary and Multiple Stars (Cambridge University Press)

Elitzur, M., Brown, J. A., \& Johnson, H. R. 1989, ApJ, 341, L95

Frankowski, A. 2009, in Asymmetrical planetary nebulae IV, ed. R. L. M. Corradi, A. Manchado, \& N. Soker (I.A.C. electronic publication), 501

Frankowski, A., \& Jorissen, A. 2007, Baltic Astron., 16, 104

Frankowski, A., \& Tylenda, R. 2001, A\&A, 367, 513

Frankowski, A., Famaey, B., van Eck, S., et al. 2009, A\&A, 498, 479

Friend, D. B., \& Castor, J. I. 1982, ApJ, 261, 293

Gail, H. P., \& Sedlmayr, E. 1987, A\&A, 171, 197

Gayley, K. G. 1995, ApJ, 454, 410

Glatzel, W., Kiriakidis, M., \& Fricke, K. J. 1993, MNRAS, 262, L7

Gunn, J. E., \& Griffin, R. F. 1979, AJ, 84, 752

Gustafsson, B., Edvardsson, B., Eriksson, K., et al. 2008, A\&A, 486, 951

Hamann, W.-R. 1993, Space Sci. Rev., 66, 237

Holzer, T. E., \& MacGregor, K. B. 1985, in Mass Loss from Red Giants, ed. M. Morris, \& B. Zuckerman, Astrophysics and Space Science Library, 117, 229

Howarth, I. D. 1997, The Observatory, 117, 335

Howarth, I. D., \& Smith, K. C. 2001, MNRAS, 327, 353

Huang, R. Q., \& Taam, R. E. 1990, A\&A, 236, 107

Hui-Bon-Hoa, A., LeBlanc, F., Hauschildt, P., \& Baron, E. 2001, A\&A, 377, 175

Jorgensen, U. G., \& Johnson, H. R. 1992, A\&A, 265, 168

Jorissen, A. 2003, in Asymptotic Giant Branch Stars, ed. H. Habing, \& H. Olofsson (New York: Springer Verlag), 461

Kondo, Y., \& McCluskey, G. E. 1976, in Structure and Evolution of Close Binary Systems, ed. P. Eggleton, S. Mitton, \& J. Whelan, IAU Symp., 73, 277

Kudritzki, R.-P., \& Puls, J. 2000, ARA\&A, 38, 613
Lamers, H. J. G. L. M. 1997, in Lecture Notes in Physics, Stellar Atmospheres: Theory and Observations (Berlin: Springer Verlag) ed. J. P. de Greve, R. Blomme, \& H. Hensberge, 69, 497

Langer, N. 1989, A\&A, 210, 93

Lemke, M. 1990, A\&A, 240, 331

Maeder, A., \& Meynet, G. 2000, A\&A, 361, 159

Mastrodemos, N., \& Morris, M. 1998, ApJ, 497, 303

Mastrodemos, N., \& Morris, M. 1999, ApJ, 523, 357

Michaud, G., \& Charland, Y. 1986, ApJ, 311, 326

Michaud, G., Tarasick, D., Charland, Y., \& Pelletier, C. 1983, ApJ, 269, 239

Mikołajewska, J. 2007, Baltic Astron., 16, 1

Moffat, A. F. J., \& Marchenko, S. V. 1996, A\&A, 305, L29

Mürset, U., \& Schmid, H. M. 1999, A\&AS, 137, 473

Nugis, T., \& Lamers, H. J. G. L. M. 2000, A\&A, 360, 227

Owocki, S. 2004, in Evolution of Massive Stars, Mass Loss and Winds, ed. M. Heydari-Malayeri, P. Stee, \& J.-P. Zahn, EAS Pub. Ser., 13, 163

Owocki, S. 2007, in Massive Stars in Interactive Binaries, ed. N. St.-Louis, \& A. F. J. Moffat, ASP Conf. Ser., 367, 233

Owocki, S. P., \& Gayley, K. G. 1999, in Wolf-Rayet Phenomena in Massive Stars and Starburst Galaxies, ed. K. A. van der Hucht, G. Koenigsberger, \& P. R. J. Eenens, IAU Symp., 193, 157

Pauldrach, A., Puls, J., \& Kudritzki, R. P. 1986, A\&A, 164, 86

Phillips, S. N., \& Podsiadlowski, P. 2002, MNRAS, 337, 431

Puls, J., Springmann, U., \& Lennon, M. 2000, A\&AS, 141, 23

Rafert, J. B., \& Twigg, L. W. 1980, MNRAS, 193, 79

Reimers, D. 1975, Mem. Soc. Roy. Sci. Liège, 6th Ser., 8

Ritter, H. 1996, in Evolutionary Processes in Binary Stars, ed. R. A. M. J. Wijers,

M. B. Davies, \& C. A. Tout (Dordrecht: Kluwer), 223

Sandin, C. 2008, MNRAS, 385, 215

Sandin, C., \& Höfner, S. 2003, A\&A, 398, 253

Schatzman, E. L., Praderie, F., \& King, A. R. 1993, The Stars, The Stars. Schatzman, Evry L., Praderie, Francoise (Berlin Heidelberg: Springer-Verlag), 402

Schröder, K.-P., Wachter, A., \& Winters, J. M. 2003, A\&A, 398, 229

Schuerman, D. W. 1972, Ap\&SS, 19, 351

Shimada, M. R., Ito, M., Hirata, B., \& Horaguchi, T. 1994, in Pulsation; Rotation; and Mass Loss in Early-Type Stars, ed. L. A. Balona, H. F. Henrichs, \& J. M. Le Contel, IAU Symp., 162, 487

Soberman, G. E., Phinney, E. S., \& van den Heuvel, E. P. J. 1997, A\&A, 327, 620

Sytov, A. Y., Bisikalo, D., Kaigorodov, P., \& Boyarchuk, A. 2009, Astron. Rep., 53, 223

Theuns, T., \& Jorissen, A. 1993, MNRAS, 265, 946

Theuns, T., Boffin, H. M. J., \& Jorissen, A. 1996, MNRAS, 280, 1264

Tout, C. A., \& Eggleton, P. P. 1988, MNRAS, 231, 823

Vanbeveren, D. 1977, A\&A, 54, 877

Vanbeveren, D. 1978, Ap\&SS, 57, 41

Wachter, A., Schröder, K.-P., Winters, J. M., Arndt, T. U., \& Sedlmayr, E. 2002, A\&A, 384, 452

Willson, L. A. 2000, ARA\&A, 38, 573

Zhou, H.-N., \& Leung, K.-C. 1988, Ap\&SS, 141, 257 